\title{
. The Effect of Nuclear-Quadrupole Coupling in the Laser-Induced Alignment of Molecules
}

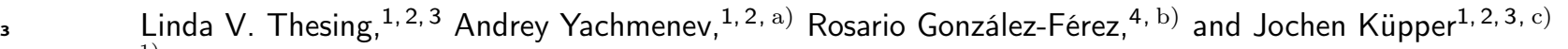 \\ ${ }^{1)}$ Center for Free-Electron Laser Science, Deutsches Elektronen-Synchrotron DESY, Notkestrasse 85, 226077 Hamburg, \\ Germany \\ 2) The Hamburg Center for Ultrafast Imaging, Universität Hamburg, Luruper Chaussee 149, 22761 Hamburg, Germany \\ 3) Department of Physics, Universität Hamburg, Luruper Chaussee 149, 22761 Hamburg, Germany \\ ${ }^{4)}$ Instituto Carlos I de Física Teórica y Computacional and Departamento de Física Atómica, Molecular y Nuclear, \\ Universidad de Granada, 18071 Granada, Spain
}

(Dated: 6 February 2020)

\begin{abstract}
We present a theoretical study of the time-dependent laser alignment of molecules taking into account the hyperfine coupling due to nuclear-quadrupole interactions. The coupling of nuclear spins to the overall angular momentum of molecules significantly influences their rotational dynamics. Here, we systematically analyze the impact of the nuclear-quadrupole coupling on the rotational dynamics of the linear $\mathrm{I}_{2}$ and the asymmetric-top diiodobenzene molecule induced by external laser fields. We explore different regimes of pulse shapes and laser-pulse intensities and detail under which conditions the quadrupole coupling cannot be neglected in the description of the laser alignment of molecules.
\end{abstract}

\section{I. Introduction}

12 Controlling the rotational motion of molecules with ex13 ternal electric fields is among the most interesting goals in 14 physical chemistry. The simplest approach to theoretically 15 describe this field-induced control relies on applying the 16 rigid-rotor approximation. For many molecular species, 17 this approach has been shown to be sufficient, 15 even for 18 some floppy molecules. ${ }^{6}$ However, the coupling of the over19 all angular momentum to additional angular momenta 2o or internal rotations cannot be neglected under certain 21 circumstances. For instance, it has been shown that cou22 pling of nuclear spins and the overall angular momentum 23 can have a significant impact on the rotational dynamics 24 on experimentally relevant timescales.

25 Fixing molecules in space, i. e., aligning and orienting 26 them, 16 is of particular interest among rotational con27 trol schemes as it reduces the blurring of experimental 28 observables caused by averaging over the random orienta29 tions and allows to obtain information in the molecular so frame 21 Molecules can be aligned by subjecting them 31 to nonresonant laser fields. If the laser pulse is switched 32 on slowly compared to the molecular rotational period, 33 adiabatic alignment is achieved. On the other hand, by us34 ing short laser pulses, coherent superpositions of field-free 35 rotational states are created. The wave packets rephase 36 periodically and show revivals of the alignment in field-free 37 space 122 Such coherent superpositions can also be ob38 tained by shaped laser pulses which are turned off rapidly 39 compared to the rotational period of the molecule. 25

40 Recently, it has been shown that the impulsive align41 ment of $\mathrm{I}_{2}$ molecules can only be described accurately if

\footnotetext{
a) andrey.yachmenev@cfel.de

b) rogonzal@ugr.es

c) Email: jochen.kuepper@cfel.de

website: https://www.controlled-molecule-imaging.org
}

42 the nuclear-quadrupole coupling is taken into account 30 ${ }_{43}$ We previously developed a generalized methodology to 44 describe the rovibrational dynamics of molecules includ45 ing nuclear-quadrupole interactions ${ }^{31}$ Here, we system46 atically analyze the impact of this coupling in different 47 existing techniques for laser alignment of prototypical 48 linear and asymmetric top molecules using $\mathrm{I}_{2}$ and 1,449 diiodobenzene as examples. Our results thereby serve as so a guideline to understand under which circumstances the 51 quadrupole coupling has a significant influence on the 52 rational dynamics.

\section{II. Theoretical Description}

$54 \quad$ In $\mathrm{I}_{2}$ and 1,4-diiodobenzene (DIB), the interaction be55 tween the nuclear quadruple moments of two equivalent 56 iodine ${ }^{127}$ I nuclei and the electric field gradients, arising 57 from the charge distributions of the surrounding nuclei 58 and electrons, lead to the well known hyperfine splittings 59 of the rotational energy levels. Each rotational level of 60 the molecule is thus split into a maximum of 36 sub-levels, 61 labeled by the quantum number $F$ of the total angular 62 momentum operator $\boldsymbol{F}=\boldsymbol{J}+\boldsymbol{I}$, where $\boldsymbol{J}$ is the rotational 63 angular momentum neglecting the spin, which for the 64 molecules considered here is the angular momentum of ${ }_{65}$ overall rotation. Here, $\boldsymbol{I}=\boldsymbol{I}_{1}+\boldsymbol{I}_{2}$ is the collective nuclear 66 spin angular momentum operator with $I_{1}=I_{2}=5 / 2$ and 67 thus $0 \leq I \leq 5$.

${ }_{68}$ The theoretical model for the nuclear-quadrupole inter69 actions has been described before ${ }^{31}$ Briefly, within the 7o Born-Oppenheimer and semirigid-rotor approximations, 71 the field-free Hamiltonian can be written as

$$
H_{\mathrm{mol}}=H_{\mathrm{rot}}+\sum_{l=1,2} \boldsymbol{V}(l) \cdot \boldsymbol{Q}(l),
$$

72 where $H_{\text {rot }}$ is the semirigid-rotor Hamiltonian. The rota73 tional constant of $\mathrm{I}_{2}$ was experimentally determined to 
${ }_{74} B=1118.63 \mathrm{MHz}$ [30 For DIB, the rotational constants 126 such as $K_{a}$ and $K_{c}$ for DIB. For I ${ }_{2}$, we took into account ${ }_{75} B_{z}=A=5712.768 \mathrm{MHz}, B_{y}=B=159.017 \mathrm{MHz}$, and ${ }_{127}$ the symmetry requirement that $J$ and $I$ have to be either ${ }_{76} B_{x}=C=154.710 \mathrm{MHz}$ were obtained from a geome- 128 both even or both odd, 45 while for DIB all combinations 77 try optimization using density functional theory (DFT) 129 of basis states are allowed. The rotational states $|J, w\rangle$ 78 with the B3LYP functional and the def2-QZVPP ba- 1 so were obtained as linear combinations of symmetric top

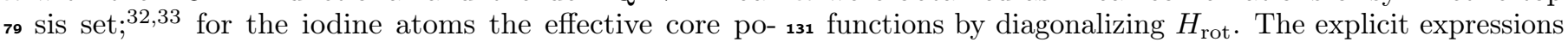
so tential def2-ECP was used ${ }^{34}$ All electronic structure ${ }_{132}$ for the matrix elements of the quadrupole coupling Hamil81 calculations employed the quantum-chemistry package 133 tonian and various multipole Cartesian tensor operators ${ }_{82}$ ORCA $\sqrt[35 \mid 36]{36}$ The molecule-fixed frame (MFF), $x, y, z$, is ${ }_{134}$ can be found elsewhere ${ }^{31 / 46 / 47}$ To obtain the matrix rep83 defined by the principal axes of inertia. In the second 135 resentation of the interaction Hamiltonian, the matrix $\mathbf{8 4}$ term, $\boldsymbol{Q}(l)$ is the nuclear-quadrupole tensor of the $l$-th $\mathbf{1 3 6}$ elements were first set up in the coupled basis $\frac{46}{}$ and then 85 iodine nucleus and $\boldsymbol{V}(l)$ is the electric-field-gradient ten- 137 transformed to the field-free eigenbasis ${ }^{31}$ The alignment 86 sor at the instantaneous position of the corresponding ${ }_{138}$ is quantified by $\left\langle\cos ^{2} \theta\right\rangle$, with the Euler angle $\theta$ between 87 nucleus. Due to the symmetry of DIB, the electric-field- 139 the molecule-fixed $z$ and the laboratory-fixed $Z$ axes.

s8 gradient (EFG) tensors on the two iodine centers are

s9 equal to each other with nonzero elements only on the

9o diagonal $V_{x x}=-5.5879$ a.u., $V_{y y}=-6.2295$ a.u. and ${ }_{140}$ III. Results and Discussion

${ }_{91} V_{z z}=11.8174$ a. u.. The nuclear quadrupole moment for ${ }_{92}{ }^{127} \mathrm{I}$ is $Q=-696 \mathrm{mb} \cdot \frac{37}{37}$ For $\mathrm{I}_{2}$, instead of computing the 93 EFG tensors we used the experimental nuclear-quadrupole 94 coupling constant $\chi_{z z}=e Q V_{z z}=-2.45258 \mathrm{GHz} \stackrel{[38}{\text { with }}$ 95 the elementary charge $e$.

96 The interaction of a molecule with a nonresonant laser 97 field, linearly polarized along the laboratory-fixed $Z$ axis, $\mathbf{9 8}$ is given by

$$
H_{\text {las }}(t)=-\frac{I(t)}{2 \varepsilon_{0} c} \alpha_{Z Z}
$$

141 Adiabatic alignment We considered a linearly po142 larized laser pulse with a Gaussian envelope $I(t)=$ ${ }_{143} I_{0} \exp \left(-4 \ln 2 t^{2} / \tau_{\mathrm{FWHM}}^{2}\right)$, where $\tau_{\mathrm{FWHM}}=8 \mathrm{~ns}$ and ${ }_{144} I_{0}=2 \times 10^{11} \mathrm{~W} / \mathrm{cm}^{2}$. For the rotational temperature ${ }_{145} \mathrm{~T}_{\text {rot }}=0 \mathrm{~K}$, we assumed that all hyperfine states cor146 responding to the rotational ground state $J=0$ were 147 populated according to their statistical weights. For $\mathrm{I}_{2}$, 148 the spin-rotational states with even $I$ have equal weights 149 while the ones with odd $I$ have zero weights $\stackrel{45}{\text { For DIB, }}$ 99 where $\alpha_{Z Z}$ is the element of the polarizability tensor of ${ }_{\mathbf{1 5 0}}$ the Hydrogen nuclear-spin functions result in weights of 7 100 the molecule along the laser polarization axis. The po- ${ }_{151}$ (3) for even (odd) I; see Appendix A The adiabatic align101 larizabilities defined in the laboratory frame are directly $\mathbf{1 5 2}$ ment with nuclear-quadrupole coupling was computed by 102 transformed to the molecular frame $\alpha_{i j}(i, j=x, y, z) \cdot{ }_{153}^{152}$ averaging over individually obtained alignment results for 103 For both, $\mathrm{I}_{2}$ and DIB, the polarizability tensor $\alpha_{i j}$ is 154 the 15 (36) initial nuclear-spin states of $\mathrm{I}_{2}$ (DIB).

104 diagonal in the inertial frame. For $\mathrm{I}_{2}$, we used the values ${ }_{155}$ The interaction of the external electric field with the ${ }_{105} \alpha_{x x}=\alpha_{y y}=7.94 \AA^{3}$ and $\alpha_{z z}=13.96 \AA^{3}{ }^{40}$ For DIB, ${ }_{156}$ polarizability of the molecules is much stronger than the 106 calculated values of $\alpha_{x x}=11.307 \AA^{3}, \alpha_{y y}=16.676 \AA^{3}$, 107 and $\alpha_{z z}=32.667 \AA^{3}$ were used 41 . Calculations of the 108 EFG and polarizability tensors for the DIB molecule were 109 carried out at the DFT/B3LYP level of theory using the 110 all-electron scalar relativistic Douglas-Kroll-Hess Hamil111 tonian ${ }^{42}$ with the DKH-def2-TZVP basis set. $13 \mid 44$

${ }_{112}$ To study the rotational dynamics of $\mathrm{I}_{2}$ and DIB, we 113 solved the time-dependent Schrödinger equation (TDSE) 114 for the full Hamiltonian

$$
H(t)=H_{\mathrm{mol}}+H_{\mathrm{las}}(t) .
$$

157 quadrupole-coupling interaction, resulting in the decou158 pling of the nuclear spins from the overall-rotation angular 159 momentum. As a result, the influence of the nuclear160 quadrupole coupling on the adiabatic alignment is negli161 gible for both molecules. At the peak intensity, we thus 162 obtained $\left\langle\cos ^{2} \theta\right\rangle=0.946$ and $\left\langle\cos ^{2} \theta\right\rangle=0.988$ for $\mathrm{I}_{2}$ 163 and DIB, respectively, both, including and neglecting the 164 quadrupole coupling.

165 Impulsive alignment We analyzed the impulsive (3) 166 alignment induced by short $\left(\tau_{\mathrm{FWHM}}=1 \mathrm{ps}\right)$ nonresonant 167 linearly polarized laser pulses with Gaussian envelopes. 115 The time-dependent wave function was built from a super- ${ }_{168}$ We compared the post-pulse dynamics including and ne116 position of the field-free spin-rotational eigenfunctions of 169 glecting the nuclear-quadrupole coupling for the rotational ${ }_{117} H_{\text {mol }}$. The time-dependent coefficients were determined 170 gound states, i. e., $\mathrm{T}_{\text {rot }}=0 \mathrm{~K}$. To allow for a better com118 by numerical solution of the TDSE using the split-operator $\mathbf{1 7 1}$ parison of the alignment with and without the coupling, 119 method using RichMol ${ }^{39}$ To obtain the field-free eigen- 172 we used initial states with well defined rotational quantum 120 functions, we solved the time-independent Schrödinger 173 numbers, i. e., uncoupled states $\left|I M_{I}\right\rangle|00\rangle\left(\left|I M_{I}\right\rangle\left|0_{00} 0\right\rangle\right)$ 121 equation for the Hamiltonian $H_{\text {mol }}$. The matrix represen- $\mathbf{1 7 4}$ for $\mathrm{I}_{2}$ (DIB), and averaged over the results for the dif122 tation of $H_{\text {mol }}$ was constructed in a symmetry-adapted 175 ferent spin isomers, see above. We point out that the 123 coupled basis $|F, J, I, w\rangle$ of the rotational wave functions 176 corresponding field-free eigenstates have contributions of $124|J, w\rangle$ and the nuclear-spin functions $|I\rangle$. Here, $w$ repre- $177 \quad J>0$ rotational states, which might lead to additional 125 sents additional rotational (pseudo) quantum numbers, $\mathbf{1 7 8}$ differences in the dynamics. To solve the TDSE, we 
$\mathrm{I}_{2}$

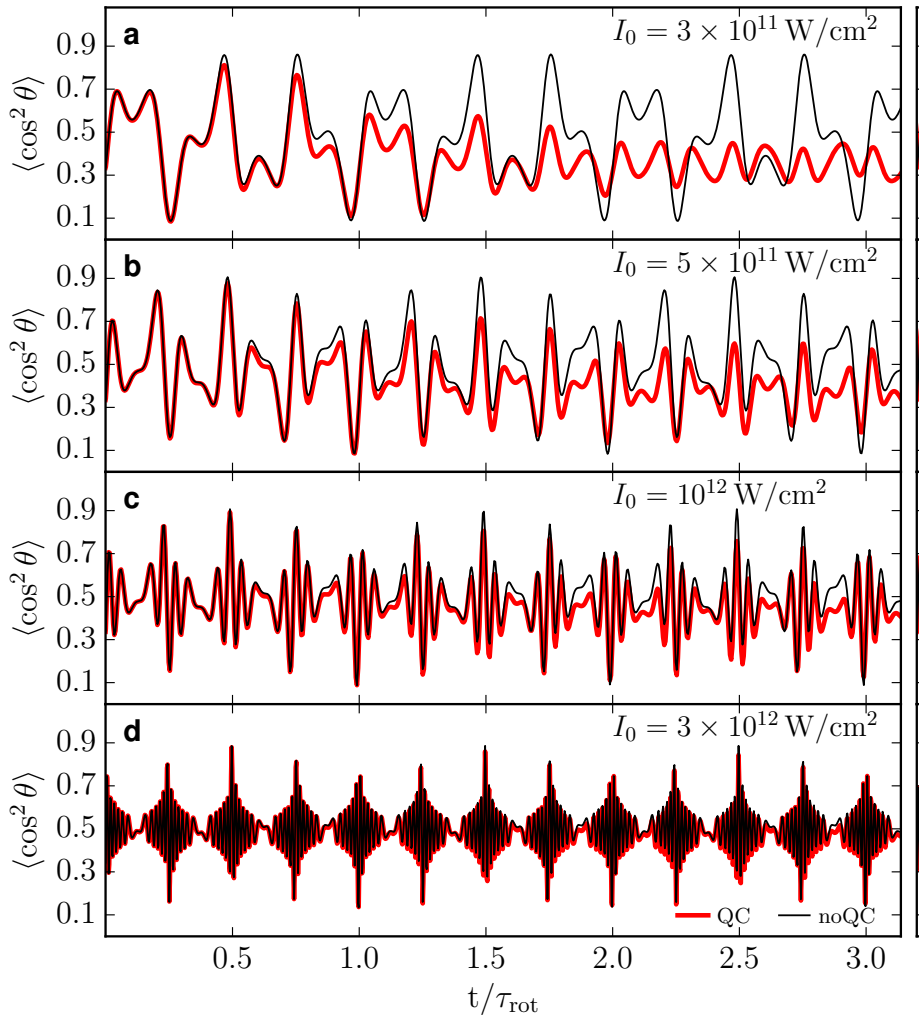

DIB

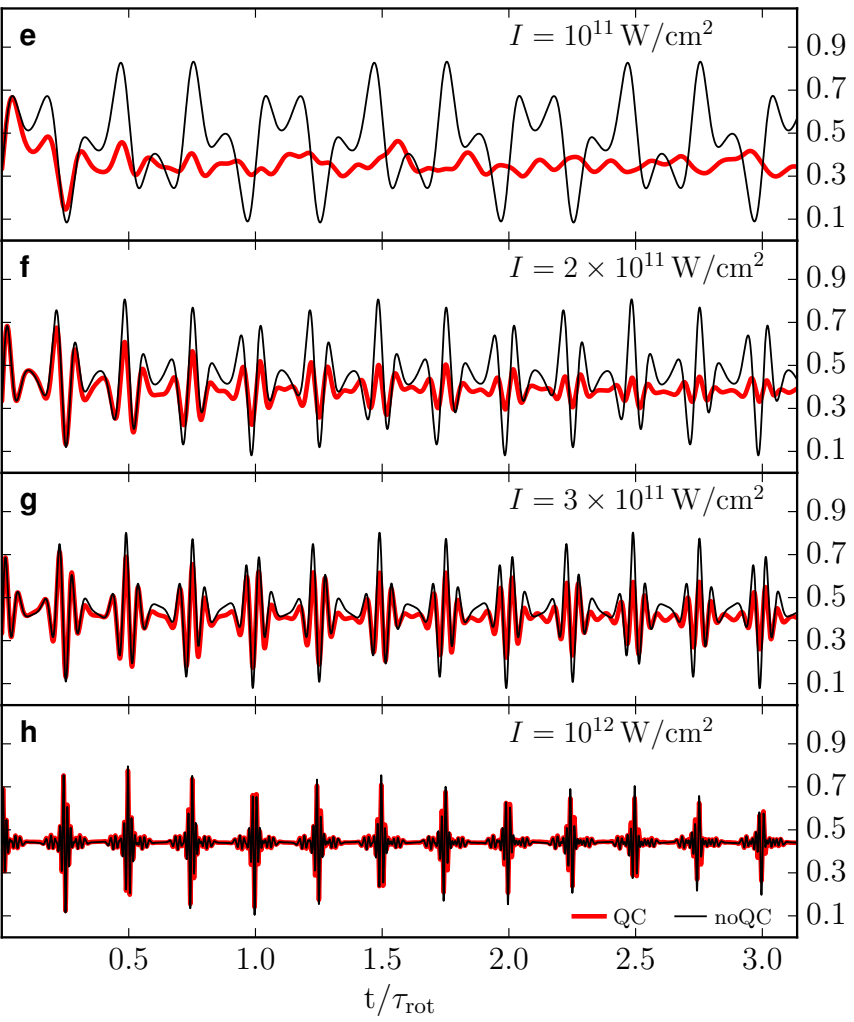

FIG. 1. Impulsive alignment induced by laser pulses with $\tau_{\mathrm{FWHM}}=1 \mathrm{ps}$ for $(\mathrm{a}-\mathrm{d}) \mathrm{I}_{2}$ and $(\mathrm{e}-\mathrm{h})$ DIB including (QC) and neglecting the nuclear-quadrupole coupling (noQC). The expectation value $\left\langle\cos ^{2} \theta\right\rangle$ is shown as a function of $t / \tau_{\text {rot }}$, where $\tau_{\text {rot }}$ is the rotation period of the molecules. For each row, the laser intensities were chosen to create rotational wave packets involving similar distributions of $J$ values for both molecules when the quadrupole coupling is neglected.

179 projected each initial state onto the field-free eigenbasis. ${ }_{203}$ DIB. However, during the short pulse the quadrupole in180 Fig. 1 shows the post-pulse alignment for $(\mathrm{a}-\mathrm{d}) \mathrm{I}_{2}$ for 204 teraction plays a negligible role for both molecules due to 181 intensities $3 \times 10^{11} \mathrm{~W} / \mathrm{cm}^{2}<I_{0}<3 \times 10^{12} \mathrm{~W} / \mathrm{cm}^{2}$ and ${ }^{205}$ a decoupling of the nuclear spin and overall-rotation an${ }_{182}(\mathrm{e}-\mathrm{h})$ DIB for intensities $10^{11} \mathrm{~W} / \mathrm{cm}^{2}<I_{0}<10^{12} \mathrm{~W} / \mathrm{cm}^{2}{ }^{206}$ gular momenta. The laser field only affects the rotational ${ }_{183}$ as a function of time in units of the rotational periods, ${ }^{207}$ part of the wave packet, leaving the nuclear-spin quanta ${ }_{184} \tau_{\text {rot }}=446.98$ ps and $\tau_{\text {rot }}=3187.48$ ps for $\mathrm{I}_{2}$ and DIB, ${ }^{208}$ unchanged. As a consequence, the alignment traces with 185 respectively. The post-pulse alignment simulated without ${ }^{209}$ and without the quadrupole coupling are very similar to 186 the quadrupole interaction shows typical revival structures ${ }^{210}$ each other directly after the laser pulse. This also holds ${ }_{187}$ for rotational wave packets induced by short laser pulses. ${ }^{211}$ for higher laser intensities.

${ }_{188}$ For the smallest intensities, Fig. 1 a, e the field-dressed 189 dynamics is dominated by a few low-energy rotational 190 states and $\left\langle\cos ^{2} \theta\right\rangle$ oscillates with the period $\tau_{\text {rot }}$. For DIB 191 and $I_{0}=10^{11} \mathrm{~W} / \mathrm{cm}^{2}$, contributions of rotational states 192 with $K_{a}>0$ are negligible and the post-pulse dynamics is 193 similar to the one of the linear molecule $\mathrm{I}_{2}$. With increas194 ing intensity $I_{0}$, more highly excited rotational states are 195 involved in the dynamics. The rotational dynamics of DIB 196 in Fig. $1 \mathrm{~h}$ shows a decrease of the peak alignment over 197 time resulting from the asymmetry splitting of rotational 198 states with $K_{a}>0.2148$

${ }^{212}$ For DIB, the alignment then decreases quickly after the 213 pulse, while for $\mathrm{I}_{2}$ the rotational dynamics starts to differ ${ }_{214}$ at $t \approx \tau_{\text {rot }} / 2$. This decrease in the field-free alignment ${ }_{215}$ for small intensities can be rationalized in terms of the ${ }_{216}$ hyperfine energy levels of the Hamiltonian (1). For the ${ }_{217}$ low-energy rotational states, which contribute most to the ${ }_{218}$ dynamics in Fig. $1 \mathrm{a}$, e, the hyperfine-splitting patterns 219 depend strongly on $J$. These irregular patterns introduce 220 incommensurate frequencies that lead to a dephasing of 221 the wave packet, thus preventing strong revivals of the 222 alignment. In contrast to $\mathrm{I}_{2}$, for DIB the energy differences 199 The effect of the nuclear-quadrupole coupling on the 223 of hyperfine components within the low-energy rotational 200 alignment depends strongly on the laser intensity. For 224 states are similar to the energy differences between the 201 the low intensities in Fig. $1 \mathrm{a}$, e it is strongest and the 225 rotational levels themselves and strong inter- $J$ coupling is 202 field-free alignment decreases over time for both $\mathrm{I}_{2}$ and 226 observed in the spin-rotational states. As a consequence, 
227 the alignment of DIB is affected much more strongly and 228 faster, with respect to the rotational timescale, than it is 229 for $\mathrm{I}_{2}$.

230 The influence of the quadrupole coupling can also be 231 interpreted using a classical picture. The precession of $\boldsymbol{I}$ 232 and $\boldsymbol{J}$ around $\boldsymbol{F}$ results in a variation of their projections ${ }_{233} M_{J}$ and $M_{I}$, leading to a decrease of the alignment. In 234 addition, the magnitudes $|\boldsymbol{I}|$ and $|\boldsymbol{J}|$ are changed over 235 time by the quadrupole coupling, further affecting the 236 rotational dynamics. Note that for $\mathrm{I}_{2}$, the changes in $|\boldsymbol{J}|$ 237 are very small but a change in $|\boldsymbol{I}|$ stills affects the spatial 238 orientation of $\boldsymbol{J}$, since the total angular momentum $\boldsymbol{F}$ is 239 preserved.

240 As the laser intensity was risen, Fig. $1 \mathrm{~b}-\mathrm{d}, \mathrm{f}-\mathrm{h}$, the 241 influence of the quadrupole interaction diminished for 242 both molecules. In Fig. $1 \mathrm{~d}, \mathrm{~h}$, minor differences can only 243 be observed after the first rotational period. For these 244 strong fields, highly excited states with up to $J \approx 44$ 245 (and $K_{a} \approx 10$ for DIB) dominate the post-pulse dynam246 ics, for which the hyperfine patterns become increasingly 247 uniform. $\frac{49 \sqrt[50]{0}}{}$ For these large $J$, the matrix elements of $248 \cos ^{2} \theta$ that contribute significantly to the alignment are 249 those between field-free eigenstates with $\Delta F=\Delta J$ and 250 the same nuclear-spin contributions. Since the hyperfine 251 energy shifts are approximately the same for these states, 252 their energy gaps are very similar to those between the 253 corresponding rotational levels. As a result, we observe 254 only a very weak dephasing in Fig. $1 \mathrm{~d}, \mathrm{~h}$.

255 If field-free eigenstates are used as initial states, the 286 reaches the same value as during the pulse, $\left\langle\cos ^{2} \theta\right\rangle=$ 256 averaged alignment results for $\mathrm{I}_{2}$ do not differ significantly $\mathbf{2 8 7} 0.96$. Analogous behavior was observed for DIB with 257 from the result in Fig. 1] For DIB on the other hand, we ${ }^{288}\left\langle\cos ^{2} \theta\right\rangle=0.98$. Note that such high degrees of the post258 observe small deviations originating from a considerable $\mathbf{2 8 9}$ pulse alignment for DIB are generally possible because 259 mixing of different $J$-states in the hyperfine eigenstates, 290 the molecule is a near symmetric-top with only small 260 modifying the initial rotational wave function. The impact $2 \mathbf{2 1}$ populations of states with $K_{a}>0$, effectively reducing $\mathbf{2 6 1}$ of the quadrupole interaction is, however, qualitatively $\mathbf{2 9 2}$ the dynamcis to that of a linear rotor. Generally, the peak 262 the same. The different sets of initial states can only be 293 alignment of asymmetric top molecules at the full revival 263 considered equivalent when the result is averaged over all $2 \mathbf{2 9}$ does not reach the same value as at the peak intensity of 264 different spin isomers and $M$-states for a given rotational 295 the truncated pulse ${ }^{[27 \mid 29}$

265 level. Individual eigenstates are in general linear combi- 296 For both molecules, the dephasing due to the nuclear266 nations of uncoupled states with different values of $I, M_{I} 297$ quadrupole coupling is comparable to the impulsive align267 and $M_{J}$, even if the coupling of different $J$-states by the 298 ment case with intermediate intensities, see Fig. 1 b, $\mathrm{f}$ For 268 quadrupole interactions is neglected.

269 Truncated pulse alignment For asymmetric top 301 during the pulse, where the dynamics shows nonadiabatic 270 molecules, truncated laser pulses typically allow one to $\mathbf{3 0 2}$ behavior. The alignment then starts to slightly deviate 271 obtain larger degrees of field-free alignment than short 303 from the quadrupole-free results directly after the trun272 laser pulses, taking advantage of the initial adiabatic align- $\mathbf{3 0 4}$ cation of the laser field. By including the coupling, the ${ }_{273}$ ment 2529 Here, we consider a laser pulse with a rising 305 strongest peak alignment was observed at the full revival 274 and falling edge, both with a Gaussian shape correspond- $306 t=\tau_{\text {rot }}$ with $\left\langle\cos ^{2} \theta\right\rangle=0.85$ (0.84) for $\mathrm{I}_{2}$ (DIB), which is 275 ing to $\tau_{\mathrm{FWHM}}=600 \mathrm{ps}$ and $\tau_{\mathrm{FWHM}}=2 \mathrm{ps}$, respectively, $\mathbf{3 0 7}$ larger than the peak alignment obtained in the impulsive 276 and a peak intensity of $I_{0}=5 \times 10^{11} \mathrm{~W} / \mathrm{cm}^{2}$

зов regime, see Fig. $1 \mathrm{~d}, \mathrm{~h}$. However, the enhancement of 277 The alignment results for $\mathrm{I}_{2}$ and DIB as a function of 309 the post-pulse alignment by using truncated laser pulses ${ }_{278} t / \tau_{\text {rot }}$ are shown in Fig. $2 \mathrm{a}, \mathrm{b}$, respectively. For both 310 instead of impulsive-kick pulses was much smaller than 279 molecules, strong alignment was reached before the cut- $\mathbf{3 1}$ without the quadrupole interaction.

$\mathbf{2 8 0}$ off of the laser field with $\left\langle\cos ^{2} \theta\right\rangle$ close to 1 . As in the

281 adiabatic regime, the effect of the quadrupole coupling on 312 Post-pulse dynamics of excited rotational 282 the degree of alignment is very weak in the presence of the 313 states While state-selected molecular beams $20|24| 51,55$ $2 \mathbf{2 8}$ field. After the laser field is switched off, the quadrupole- $\mathbf{3 1 4}$ or ultracold-molecules techniques $56 \mid 57$ can produce near- 


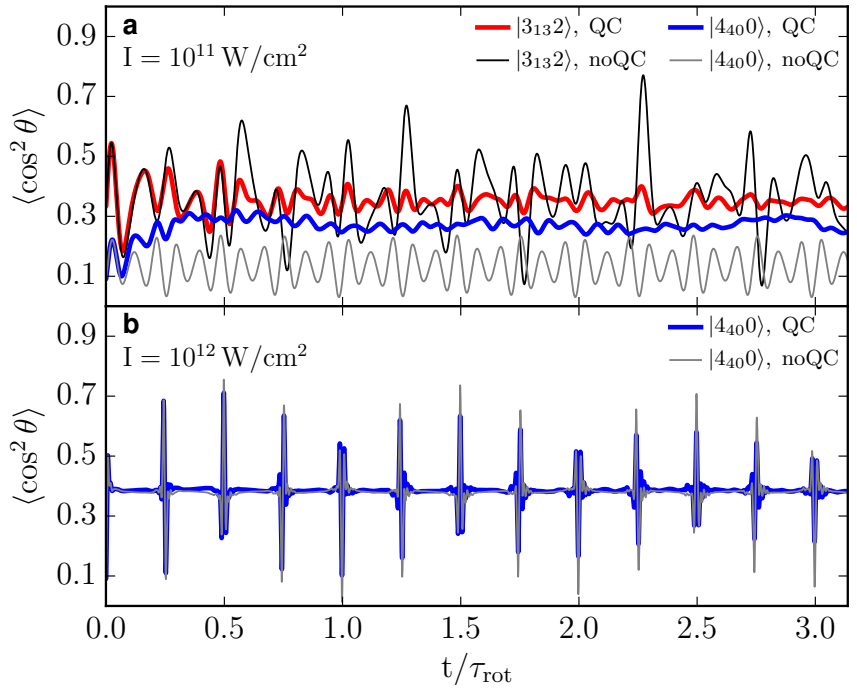

FIG. 3. Impulsive alignment for the initial states $\left|3{ }_{13} 2\right\rangle$ and $\left|4_{40} 0\right\rangle$ of DIB including the quadrupole coupling (QC) and neglecting it (noQC) as a function of $t / \tau_{\text {rot }}$. The duration of the laser pulse is $\tau_{\mathrm{FWHM}}=1 \mathrm{ps}$ and (a) $I_{0}=1 \times 10^{11} \mathrm{~W} / \mathrm{cm}^{2}$ and (b) $I_{0}=1 \times 10^{12} \mathrm{~W} / \mathrm{cm}^{2}$.

$3150 \mathrm{~K}$ samples, this is not generally feasible, especially not ${ }_{316}$ for the heavy organic molecules discussed here. There- 3 317 fore, we investigated the impulsive-alignment dynamics 318 for several initially excited states of $\mathrm{I}_{2}$ and DIB. Since ${ }^{3}$ 319 comparable results were obtained for both molecules, we 320 focus our analysis on DIB, namely the rotational states ${ }^{37}$ $321\left|J_{K_{a} K_{c}}, M_{J}\right\rangle=\left|3_{13}, 2\right\rangle$ and $\left|4_{40}, 0\right\rangle$. For both excited $\mathbf{3 7 2}$ even values of $I$. The representation generated by the 322 states, we observe a qualitatively similar influence of the 373 hydrogen nuclear-spin functions was derived $\frac{\sqrt{58}}{\text { as }} 7 A_{\mathrm{g}} \oplus$ ${ }_{323}$ quadrupole coupling as for the rotational ground state. ${ }^{374} 3 B_{1 \mathrm{~g}} \oplus 3 B_{2 \mathrm{~g}} \oplus 3 B_{3 \mathrm{~g}}$. We considered rotational states 324 For $I_{0}=1 \times 10^{11} \mathrm{~W} / \mathrm{cm}^{2},\left\langle\cos ^{2} \theta\right\rangle$ in Fig. 3 a approaches 375 having either $A_{\mathrm{g}}\left(\left|J_{K_{a} K_{c}}\right\rangle=\left|0_{00}\right\rangle\right.$ and $\left.\left|4_{40}\right\rangle\right)$ or $B_{2 \mathrm{~g}}\left(\left|3_{13}\right\rangle\right)$ $3251 / 3$ on timescales similar to the one in Fig. 1 e For the 376 symmetry. With the requirement that the total internal 326 higher intensity $I_{0}=1 \times 10^{12} \mathrm{~W} / \mathrm{cm}^{2}$, we find a more $\mathbf{3 7 7}$ wave function has to be of $B_{1 \mathrm{~g}}$ or $B_{1 \mathrm{u}}$ symmetry, we 327 significant decrease of the post-pulse alignment for the 378 obtained weights of 7 (3) for even (odd) $I$ for the former 328 initial state with $J=4, M_{J}=0$, Fig. $3 \mathrm{~b}$ than for the ro- 379 case, while for the latter all spin-rotational states have 329 tational ground state, Fig. $1 \mathrm{~h}$. This can be attributed to $\mathbf{3 8 0}$ equal weights.

ззо the populations of field-free eigenstates, which are shifted $\mathbf{3 8 1}_{\mathbf{2}}$ In the case of the diatomic $\mathrm{I}_{2}$ molecule, there are no 331 toward smaller values of $J$ for $\left|4_{40}, 0\right\rangle$. However, for other $\mathbf{3 8 2}$ additional nuclear spins leading to degeneracies of the hy332 excited states the impact of the quadrupole coupling for $\mathbf{3 8}$ perfine levels. However, in the electronic and vibrational 33 higher laser intensities can be smaller, as is the case for $\mathbf{3 8 4}$ ground state considered here, states with $J$ and $I$ having ${ }_{334}\left|3_{13} 2\right\rangle$. Thus, for a thermal ensemble of molecules, we $\mathbf{3 8 5}$ opposite parities are forbidden due to the generalized 335 expect a small influence of nuclear-quadrupole interac- 386 symmetrization postulate 45

336 tions on the post-pulse alignment induced by strong laser

337 fields. However, to accurately describe the alignment the 338 coupling cannot be fully neglected even in this regime. 387 Acknowledgments 339 Furthermore, on longer timescales even small frequency 340 shifts will lead to a significant decrease of the alignment

\section{IV. Summary and Conclusions}

388 We thank Andrey Duchko for his contributions in an 389 early part of the project.

390 This work has been supported by the Deutsche 391 Forschungsgemeinschaft (DFG) through the priority pro392 gram "Quantum Dynamics in Tailored Intense Fields" 342 In conclusion, a significant dephasing of rotational wave ${ }_{393}$ (QUTIF, SPP1840, KU 1527/3, YA 610/1) and through 34 packets was observed in the post-pulse dynamics for differ- 394 the clusters of excellence "Center for Ultrafast Imag${ }_{344}$ ent laser-field shapes and intensities. The influence on the 395 ing" (CUI, EXC 1074, ID 194651731) and "Advanced 
396 Imaging of Matter" (AIM, EXC 2056, ID 390715994). ${ }_{64}$ 397 R.G.F. gratefully acknowledges financial support by the ${ }^{465}$ 398 Spanish Project No. FIS2017-89349-P (MINECO), the ${ }^{\mathbf{4 6 6}}$ 399 Andalusian research group FQM-207, the Consejería de ${ }_{468}^{467}$ ${ }_{400}$ Conocimiento, Investigación y Universidad, Junta de An- ${ }_{460}$ 401 dalucía, and the European Regional Development Fund 470 402 (ERDF, SOMM17/6105/UGR).

\section{References} ${ }^{5}$ Trippel, S.; Mullins, T.; Müller, N. L. M.; Kienitz, J. S.; GonzálezFérez, R.; Küpper, J. Two-state wave packet for strong field-free molecular orientation. Phys. Rev. Lett. 2015, 114, 103003. Phys. Rev. A 2018, 98, 053412.

${ }^{7}$ Altkorn, R.; Zare, R. N.; Greene, C. H. Depolarization of optically prepared molecules by two randomly oriented spins. Mol. Phys. 1985, 55, 1-9.

${ }^{8}$ Yan, C.; Kummel, A. C. Effect of hyperfine depolarization upon creation and detection of alignment in free-jet expansions via selective photodissociation. J. Chem. Phys. 1993, 98, 6869-6882. ${ }^{9} \mathrm{Cool}$, T. A.; Hemmi, N. Hyperfine polarization quantum beats in cyanogen. J. Chem. Phys. 1995, 103, 3357-3366.

${ }^{10}$ Zhang, J.; Riehn, C. W.; Dulligan, M.; Wittig, C. An experimental study of HF photodissociation: Spin-orbit branching ratio and infrared alignment. J. Chem. Phys. 1996, 104, 7027-7035. Zande, W. J. Observation of fine structure and hyperfine structure depolarization in the photofragment anisotropy in triplet $\mathrm{H}_{2}$. Chem. Phys. 1997, 218, 309-323.

${ }^{12}$ Sofikitis, D.; Rubio-Lago, L.; Martin, M. R.; Ankeny Brown, D. J.; Bartlett, N. C.-M.; Alexander, A. J.; Zare, R. N.; Rakitzis, T. P. Optical control of ground-state atomic orbital alignment: $\mathrm{Cl}\left({ }^{2} P_{3 / 2}\right)$ atoms from $\operatorname{HCl}(\nu=2, J=1)$ photodissociation. J. Chem. Phys. 2007, 127, 144307.

${ }^{13}$ Bartlett, N. C.-M.; Miller, D. J.; Zare, R. N.; Alexander, A. J.; Sofikitis, D.; Rakitzis, T. P. Time-dependent depolarization of aligned HD molecules. Phys. Chem. Chem. Phys. 2009, 11, 142147.

${ }^{14}$ Bartlett, N. C.-M.; Jankunas, J.; Zare, R. N.; Harrison, J. A. Time-dependent depolarization of aligned $\mathrm{D}_{2}$ caused by hyperfine coupling. Phys. Chem. Chem. Phys. 2010, 12, 15689-15694. rotational wave-function in photodissociation of rovibrationally excited HCl molecules. J. Chem. Phys. 2017, 14\%, 013901.

${ }^{16}$ Loesch, H. J.; Remscheid, A. Brute force in molecular reaction dynamics: A novel technique for measuring steric effects. J. Chem. Phys. 1990, 93, 4779.

${ }^{17}$ Friedrich, B.; Herschbach, D. Enhanced orientation of polar molecules by combined electrostatic and nonresonant induced dipole forces. J. Chem. Phys. 1999, 111, 6157.

${ }^{18}$ Stapelfeldt, H.; Seideman, T. Colloquium: Aligning molecules with strong laser pulses. Rev. Mod. Phys. 2003, 75, 543-557.

${ }^{19}$ Spence, J. C. H.; Doak, R. B. Single molecule diffraction. Phys. Rev. Lett. 2004, 92, 198102.
${ }^{20}$ Filsinger, F.; Meijer, G.; Stapelfeldt, H.; Chapman, H.; Küpper, J. State- and conformer-selected beams of aligned and oriented molecules for ultrafast diffraction studies. Phys. Chem. Chem. Phys. 2011, 13, 2076-2087.

${ }^{21}$ Reid, K. L. Accessing the molecular frame through strong-field alignment of distributions of gas phase molecules. Phil. Trans. R. Soc. A 2018, 376, 20170158-10.

$471{ }^{22}$ Seideman, T. Revival structure of aligned rotational wave packets. 472 Phys. Rev. Lett. 1999, 83, 4971-4974.

${ }_{473}{ }^{23}$ Rosca-Pruna, F.; Vrakking, M. J. J. Revival structures in pi474 cosecond laser-induced alignemnt of $\mathrm{I}_{2}$ molecules. II. Numerical modeling. J. Chem. Phys. 2002, 116, 6579.

${ }^{24}$ Karamatskos, E. T.; Raabe, S.; Mullins, T.; Trabattoni, A.; Stammer, P.; Goldsztejn, G.; Johansen, R. R.; Długołęcki, K.; Stapelfeldt, H.; Vrakking, M. J. J.; Trippel, S.; Rouzée, A.; Küpper, J. Molecular movie of ultrafast coherent rotational dynamics of OCS. Nat. Commun. 2019, 10, 3364.

${ }^{25} \mathrm{~T}$. Seideman, On the dynamics of rotationally broad, spatially aligned wave packets. J. Chem. Phys. 2001, 115, 5965.

${ }^{26}$ Underwood, J.; Spanner, M.; Ivanov, M.; Mottershead, J.; Sussman, B.; Stolow, A. Switched wave packets: A route to nonperturbative quantum control. Phys. Rev. Lett. 2003, 90, 223001.

${ }^{27}$ Underwood, J.; Sussman, B.; Stolow, A. Field-free three dimensional molecular axis alignment. Phys. Rev. Lett. 2005, 94 143002 . molecule. Phys. Rev. A 2014, 89, 051401(R).

${ }^{28}$ Goban, A.; Minemoto, S.; Sakai, H. Laser-field-free molecular orientation. Phys. Rev. Lett. 2008, 101, 013001.

$491{ }^{29}$ Chatterley, A.; Karamatskos, E. T.; Schouder, C.; Christiansen, L.; Jörgensen, A. V.; Mullins, T.; Küpper, J.; Stapelfeldt, H. Switched wave packets with spectrally truncated chirped pulses. J. Chem. Phys. 2018, 148, 221105.

${ }^{11}$ Wouters, E. R.; Siebbeles, L. D.; Reid, K. L.; Buijsse, B.; van der

${ }^{15}$ Grygoryeva, K.; Rakovský, J.; Votava, O.; Fárník, M. Imaging of
Thomas, E. F.; Søndergaard, A. A.; Shepperson, B.; Henriksen, N. E.; Stapelfeldt, H. Hyperfine-Structure-Induced Depolarization of Impulsively Aligned $\mathrm{I}_{2}$ Molecules. Phys. Rev. Lett. 2018, 120, 163202 .

${ }^{31}$ Yachmenev, A.; Thesing, L. V.; Küpper, J. Laser-induced dynamics of molecules with strong nuclear quadrupole coupling. $J$. Chem. Phys. 2019, 151, 244118.

$502{ }^{32}$ Weigend, F.; Furche, F.; Ahlrichs, R. Gaussian basis sets of quadruple zeta valence quality for atoms $\mathrm{H}-\mathrm{Kr}$. J. Chem. Phys. 2003, 119, 12753-12762.

${ }_{05}^{33}$ Weigend, F.; Ahlrichs, R. Balanced basis sets of split valence, triple zeta valence and quadruple zeta valence quality for $\mathrm{H}$ to $\mathrm{Rn}$ : Design and assessment of accuracy. Phys. Chem. Chem. Phys. 2005, 7, 3297 .

${ }^{34}$ Peterson, K. A.; Figgen, D.; Goll, E.; Stoll, H.; Dolg, M. Systematically convergent basis sets with relativistic pseudopotentials. II. Small-core pseudopotentials and correlation consistent basis sets for the post-d group 16-18 elements. J. Chem. Phys. 2003, 119, 11113-11123.

$514{ }^{35}$ Neese, F. The ORCA program system. Wiley Interdiscip. Rev. Comput. Mol. Sci. 2011, 2, 73-78.

$5{ }^{36}$ Neese, F. Software update: the ORCA program system, version 4.0. Wiley Interdiscip. Rev. Comput. Mol. Sci. 2017, 8, e1327.

${ }^{518}{ }^{37}$ Pyykkö, P. Year-2008 nuclear quadrupole moments. Mol. Phys. 2008, 106, 1965-1974.

${ }^{38}$ Yokozeki, A.; Muenter, J. S. Laser fluorescence state selected and detected molecular beam magnetic resonance in $\mathrm{I}_{2}$. J. Chem. Phys. 1980, 72, 3796-3804.

${ }^{39}$ Owens, A.; Yachmenev, A. RichMol: A general variational approach for rovibrational molecular dynamics in external electric fields. J. Chem. Phys. 2018, 148, 124102.

${ }^{40}$ Maroulis, G.; Makris, C.; Hohm, U.; Goebel, D. Electrooptical Properties and Molecular Polarization of Iodine, $\mathrm{I}_{2}$. J. Phys. Chem. A 1997, 101, 953-956.

${ }^{41}$ For convenience and tradition, we specify polarizabilities in $\AA^{3}$, which can easily be converted to SI units as $1 \AA^{3}=10^{6} \mathrm{pm}^{3}$.

${ }^{42}$ Neese, F.; Wolf, A.; Fleig, T.; Reiher, M.; Hess, B. A. Calculation of electric-field gradients based on higher-order generalized Douglas-Kroll transformations. J. Chem. Phys. 2005, 122, 204107. 
$534{ }^{43}$ Jorge, F. E.; Neto, A. C.; Camiletti, G. G.; Machado, S. F. Con- 559

535 tracted Gaussian basis sets for Douglas-Kroll-Hess calculations: 560

536 Estimating scalar relativistic effects of some atomic and molecular 561

537 properties. J. Chem. Phys. 2009, 130, 064108.

$538{ }^{44}$ Campos, C.; Jorge, F. Triple zeta quality basis sets for atoms 56

$539 \mathrm{Rb}$ through Xe: application in $\operatorname{CCSD}(\mathrm{T})$ atomic and molecular 56

540 property calculations. Mol. Phys. 2012, 111, 167-173.

${ }_{541}{ }^{45}$ Kroll, M. Hyperfine Structure in the Visible Molecular-Iodine

542 Absorption Spectrum. Phys. Rev. Lett. 1969, 23, 631-633.

$543{ }^{46}$ Cook, R. L.; de Lucia, F. C. Application of the Theory of Irre- 568

544 ducible Tensor Operators to Molecular Hyperfine Structure. Am

545 J. Phys. 1971, 39, 1433-1454.

$546{ }^{47}$ Yachmenev, A.; Küpper, J. Communication: General variational 57155

548 of polyatomic molecules. J. Chem. Phys. 2017, 147, 141101.

$549{ }^{48}$ Holmegaard, L.; Viftrup, S. S.; Kumarappan, V.; Bisgaard, C. Z

550 Stapelfeldt, H.; Hamilton, E.; Seideman, T. Control of rotational 5

551 wave-packet dynamics in asymmetric top molecules. Phys. Rev. $\mathbf{5 7 6}$

$552 \quad A$ 2007, 75, 051403.

$553{ }^{49}$ Gordy, W.; Cook, R. L. Microwave Molecular Spectra, 3rd ed.;

554 John Wiley \& Sons: New York, NY, USA, 1984.

${ }_{555}{ }^{50}$ Kroto, H. W. Molecular Rotation Spectra; Dover Publications,

556 Inc.: Mineola, NY, USA, 2003.

$557{ }^{51}$ Hutzler, N. R.; Lu, H.-I.; Doyle, J. M. The buffer gas beam: An

558 intense, cold, and slow source for atoms and molecules. Chem.
Rev. 2012, 112, 4803-4827.

van de Meerakker, S. Y. T.; Bethlem, H. L.; Vanhaecke, N. Meijer, G. Manipulation and Control of Molecular Beams. Chem. Rev. 2012, 112, 4828-4878.

${ }^{53}$ Chang, Y.-P.; Horke, D. A.; Trippel, S.; Küpper, J. Spatiallycontrolled complex molecules and their applications. Int. Rev. Phys. Chem. 2015, 34, 557-590.

${ }^{54}$ Nielsen, J. H.; Simesen, P.; Bisgaard, C. Z.; Stapelfeldt, H.; Filsinger, F.; Friedrich, B.; Meijer, G.; Küpper, J. Stark-selected beam of ground-state OCS molecules characterized by revivals of impulsive alignment. Phys. Chem. Chem. Phys. 2011, 13 , 18971-18975.

${ }^{5}$ Horke, D. A.; Chang, Y.-P.; Długołęcki, K.; Küpper, J. Separating Para and Ortho Water. Angew. Chem. Int. Ed. 2014, 53, 1196511968.

${ }^{56}$ Ulmanis, J.; Deiglmayr, J.; Repp, M.; Wester, R.; Weidemüller, M. Ultracold Molecules Formed by Photoassociation: Heteronuclear Dimers, Inelastic Collisions, and Interactions with Ultrashort

$\mathbf{5 7 7}$ Laser Pulses. Chem. Rev. 2012, 112, 4890-4927.
${ }_{\mathbf{5 7 8}}^{57}$ Quéméner, G.; Julienne, P. S. Ultracold Molecules under Control! 579 Chem. Rev. 2012, 112, 4949-5011.

${ }_{580}^{58}$ Bunker, P. R.; Jensen, P. Molecular Symmetry and Spectroscopy 581 2nd ed.; NRC Research Press: Ottawa, Ontario, Canada, 1998. 


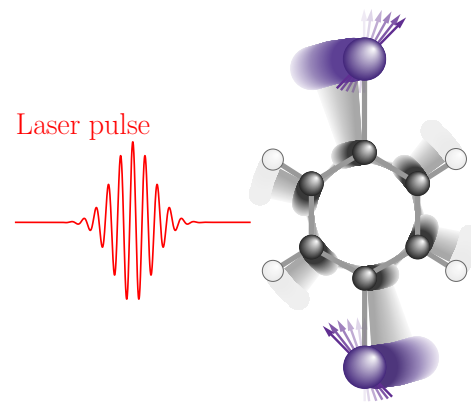

58

TOC graphic 